\title{
ON QUASINILPOTENT OPERATORS, II
}

\section{CIPRIAN FOIAŞ, IL BONG JUNG, EUNGIL KO and CARL PEARCY}

(Received 3 March 2003; revised 10 September 2003)

Communicated by G. Willis

\begin{abstract}
In this paper we continue to modify and expand a technique due to Enflo for producing nontrivial hyperinvariant subspaces for quasinilpotent operators, and thereby obtain such subspaces for some additional quasinilpotent operators on Hilbert space. We also obtain a structure theorem for a certain class of operators.
\end{abstract}

2000 Mathematics subject classification: primary 47A15.

Keywords and phrases: hyperinvariant subspaces, quasinilpotent operators.

\section{Introduction}

Let $\mathscr{H}$ be a separable, infinite dimensional, complex Hilbert space, and denote by $\mathscr{L}(\mathscr{H})$ the algebra of all bounded linear operators on $\mathscr{H}$. In [1], Per Enflo introduced a new technique involving some 'extremal vectors' for producing hyperinvariant subspaces for certain quasinilpotent operators in $\mathscr{L}(\mathscr{H})$. This technique was then modified and expanded by the present authors in [4] to produce better hyperinvariant subspaces for some additional quasinilpotent operators. This paper is a sequel to [4] in which we continue to explore the limits of Enflo's technique for producing hyperinvariant subspaces. In [4] we recovered, via this new technique, the theorem of Lomonosov [6] that if $Q$ is quasinilpotent in $\mathscr{L}(\mathscr{H})$ and the commutant

$$
\{Q\}^{\prime}=\{C \in \mathscr{L}(\mathscr{H}): C Q=Q C\}
$$

of $Q$ has the 'Pearcy-Salinas property' (see [6] and [7] for example), then $Q$ has a nontrivial hyperinvariant subspace. Herein we explore further the basic construction from [1]. This allows us to obtain the existence of hyperinvariant subspaces for

(C) 2004 Australian Mathematical Society $1446-7887 / 04 \$ A 2.00+0.00$ 
some more quasinilpotent operators, and to discuss certain structural properties of quasinilpotent operators.

\section{The basic lemmas}

For the reader's convenience, we state the basic lemmas from [4], which are themselves only minor variations of the corresponding lemmas in [1]. For proofs the reader may consult either [1] or [4]. As usual $\mathbb{N}$ will denote the set of positive integers and $\mathbb{K}$ the ideal of compact operators in $\mathscr{L}(\mathscr{H})$.

LEMMA 2.1. Suppose that $u$ and $v$ are nonzero vectors in $\mathscr{H}$ such that for every $w \in \mathscr{H}, \operatorname{Re}(u, w)<0$ implies that $\operatorname{Re}(v, w) \geq 0$. Then there exists a negative number $\gamma_{0}$ such that $v=\gamma_{0} u$.

LEMMA 2.2. Suppose $T \in \mathscr{L}(\mathscr{H}), x_{0}$ is a nonzero vector in the closure of the range of $T$, and $\varepsilon$ satisfies $0<\varepsilon<\left\|x_{0}\right\|$. Then there exists a unique nonzero vector $y_{0}=y_{0}\left(x_{0}, \varepsilon\right)$ in the set $\left\{y:\left\|T y-x_{0}\right\| \leq \varepsilon\right\}$ satisfying

(a) $\left\|y_{0}\right\|=\inf \left\{\|y\|:\left\|T y-x_{0}\right\| \leq \varepsilon\right\}$, and this vector $y_{0}$ also satisfies

(b) $\left\|T y_{0}-x_{0}\right\|=\varepsilon$.

LEMMA 2.3. Suppose $T, x_{0}, y_{0}$ and $\varepsilon$ are as in Lemma 2.2. Then there exists a negative number $\gamma_{0}$ such that $T^{*}\left(T y_{0}-x_{0}\right)=\gamma_{0} y_{0}$.

LEMMA 2.4. Suppose $T \in \mathscr{L}(\mathscr{H})$ is quasinilpotent with dense range, $x_{0}$ is a nonzero vector in $\mathscr{H}$, and $\varepsilon$ satisfies $0<\varepsilon<\left\|x_{0}\right\|$. For each $n \in \mathbb{N}$, let $y_{n}=y_{n}\left(x_{0}, \varepsilon\right)$ be the unique (nonzero) vector in the set $\left\{y:\left\|T^{n} y-x_{0}\right\| \leq \varepsilon\right\}$ satisfying (via Lemma 2.2)

(a') $\left\|y_{n}\right\|=\inf \left\{\|y\|:\left\|T^{n} y-x_{0}\right\| \leq \varepsilon\right\}$, and

(b') $\left\|T^{n} y_{n}-x_{0}\right\|=\varepsilon$.

Then there exists a subsequence $\left\{y_{n_{k}}\right\}_{k \in \mathbb{N}}$ of the sequence $\left\{y_{n}\right\}_{n \in \mathbb{N}}$ satisfying

$$
\lim _{k}\left\|y_{n_{k}}\right\| /\left\|y_{n_{k}+1}\right\|=0 \text {. }
$$

\section{Some results}

In this section we use the basic lemmas of Section 2 to produce some new results about quasinilpotent operators. The first might be called the basic construction that our modified version of Enflo's technique produces, and was unknown to us at the time [4] was written. 
THEOREM 3.1. We suppose the following:

(A) $Q$ is an arbitrary quasinilpotent operator in $\mathscr{L}(\mathscr{H})$ with dense range;

(B) $C$ is an arbitrary nonzero contraction in $\mathscr{L}(\mathscr{H})$;

(C) $x_{0}$ is an arbitrary vector in $\mathscr{H}$ such that $C x_{0} \neq 0$;

(D) $\varepsilon$ satisfies $0<\varepsilon<\left\|C x_{0}\right\|$ ( $\left.\leq\left\|x_{0}\right\|\right)$;

(E) $\left\{y_{n}\right\}_{n \in \mathbb{N}}$ is the sequence of vectors in $\mathscr{H}$ associated with $Q, x_{0}$, and $\varepsilon$ provided by Lemma 2.4, and

(F) $\left\{y_{n_{k}}\right\}_{k \in \mathbb{N}}$ is a subsequence of the sequence $\left\{y_{n}\right\}$ satisfying (1) and (without loss of generality by virtue of Lemma $2.4\left(\mathrm{~b}^{\prime}\right)$ ) also has the property that the sequences $\left\{Q^{n_{k}} y_{n_{k}}\right\}_{k \in \mathbb{N}}$ and $\left\{z_{k}:=Q^{n_{k}+1} y_{n_{k}+1}\right\}_{k \in \mathbb{N}}$ are weakly convergent to vectors $s_{0}$ and $z_{0}$, respectively.

Then,

(I) $\left\|s_{0}-x_{0}\right\| \leq \varepsilon$, which implies that $s_{0} \neq 0 \neq C s_{0}$ by virtue of (B) and (D),

(II) $0<\left\|z_{0}-x_{0}\right\| \leq \varepsilon$, which implies that $x_{0} \neq z_{0} \neq 0$ and that $Q^{*}\left(z_{0}-x_{0}\right) \neq 0$, and

(III) $\left\|y_{n_{k}}\right\|\left\|\left(Q^{n_{k}+1}\right)^{*}\left(z_{k}-x_{0}\right)\right\| \leq\left(\left\|y_{n_{k}}\right\| /\left\|y_{n_{k}+1}\right\|\right)\left(\varepsilon^{2}+\varepsilon\left\|x_{0}\right\|\right)$,

which implies, in particular, via (1) that

$$
\lim _{k}\left\|y_{n_{k}}\right\|\left\|\left(Q^{n_{k}+1}\right)^{*}\left(z_{k}-x_{0}\right)\right\|=0 .
$$

Proof. We know from Lemma $2.4\left(\mathrm{~b}^{\prime}\right)$ that $\left\|Q^{n} y_{n}-x_{0}\right\|=\varepsilon, n \in \mathbb{N}$, and since closed balls in $\mathscr{H}$ are weakly closed, (I) is established as well as the fact that $\left\|z_{0}-x_{0}\right\| \leq \varepsilon$, which shows that $z_{0} \neq 0$. We next show that $z_{0}-x_{0} \neq 0$, which will also show that $Q^{*}\left(z_{0}-x_{0}\right) \neq 0$ (since $Q$ has dense range). By the definition of the sequence $\left\{y_{n_{k}}\right\}_{k \in \mathbb{N}}$ and Lemma 2.3, we know that there exists a sequence $\left\{r_{k}\right\}_{k \in \mathbb{N}}$ of negative numbers such that

$$
\left(Q^{n_{k}+1}\right)^{*}\left(z_{k}-x_{0}\right)=r_{k} y_{n_{k}+1}, \quad k \in \mathbb{N} .
$$

Therefore,

$$
\begin{aligned}
\varepsilon^{2} & =\left\|Q^{n_{k}+1} y_{n_{k}+1}-x_{0}\right\|^{2} \\
& =\left(y_{n_{k}+1},\left(Q^{n_{k}+1}\right)^{*}\left(z_{k}-x_{0}\right)\right)-\left(x_{0}, z_{k}-x_{0}\right) \\
& =r_{k}\left\|y_{n_{k}+1}\right\|^{2}-\left(x_{0}, z_{k}-x_{0}\right), \quad k \in \mathbb{N} .
\end{aligned}
$$

Thus

$$
r_{k}\left\|y_{n_{k}+1}\right\|^{2}=\varepsilon^{2}+\left(x_{0}, z_{k}-x_{0}\right)
$$

and

$$
\left(x_{0}, z_{k}-x_{0}\right)<-\varepsilon^{2}, \quad k \in \mathbb{N} .
$$


Taking the weak limit in (6) as $k \rightarrow \infty$, we get $\left(x_{0}, z_{0}-x_{0}\right) \leq-\varepsilon^{2}$, so $z_{0}-x_{0} \neq 0$, which establishes (II). Furthermore, from (3) and (5) we see that

$$
\begin{aligned}
\left\|y_{n_{k}+1}\right\|\left\|\left(Q^{n_{k}+1}\right)^{*}\left(z_{k}-x_{0}\right)\right\| & =\left|r_{k}\right|\left\|y_{n_{k}+1}\right\|^{2} \\
& =\left|\varepsilon^{2}+\left(x_{0}, z_{k}-x_{0}\right)\right| \\
& \leq \varepsilon^{2}+\varepsilon\left\|x_{0}\right\|, \quad k \in \mathbb{N},
\end{aligned}
$$

which, together with trivial arithmetic, establishes (III).

REMARK 3.2. With the notation as in Theorem 3.1, note that the quantity

$$
\left\|y_{n_{k}}\right\|\left\|\left(Q^{n_{k}+1}\right)^{*}\left(z_{k}-x_{0}\right)\right\|
$$

in (2) is exactly the trace-norm $\left(\|\|_{1}\right)$ of the rank-one operator $y_{n_{k}} \otimes\left(Q^{n_{k}+1}\right)^{*}\left(z_{k}-x_{0}\right)$, which, of course, belongs to the trace class $\mathscr{C}_{1}(\mathscr{H})$. Since $\mathscr{C}_{1}(\mathscr{H})^{*}=\mathscr{L}(\mathscr{H})$, any rank-one operator $u \otimes v$ may be regarded as the weak*-continuous linear functional on $\mathscr{L}(\mathscr{H})$ defined by $(u \otimes v)(T)=(T u, v), T \in \mathscr{L}(\mathscr{H})$, and the norm of this linear functional is $\|u \otimes v\|_{1}=\|u\| \cdot\|v\|$. This is, of course, the basic idea behind the theory of dual algebras (see for example [2]), and together with (2) gives immediately the following.

COROLlaRY 3.3. With the notation as in Theorem 3.1, let $\left\{A_{k, m}\right\}_{k, m \in \mathbb{N}}$ and $\left\{B_{k, m}\right\}_{k, m \in \mathbb{N}}$ be any bounded doubly indexed sequences of operators from $\mathscr{L}(\mathscr{H})$. Then

$$
\lim _{k}\left(Q^{n_{k}} A_{k, m} y_{n_{k}}, Q^{*}\left(z_{k}-x_{0}\right)\right)=0, \quad m \in \mathbb{N},
$$

and

$$
\lim _{k}\left(\left(Q^{n_{k}} /\left\|Q^{n_{k}}\right\|\right) B_{k, m} Q^{n_{k}} y_{n_{k}}, Q^{*}\left(z_{k}-x_{0}\right)\right)=0, \quad m \in \mathbb{N} .
$$

To establish immediately the utility of this corollary, we deduce quickly a modest improvement, due to Pearcy-Shields [8], of the famous result of Lomonosov [5].

COROLlARY 3.4. Suppose that $Q \neq 0$ is a quasinilpotent operator in $\mathscr{L}(\mathscr{H})$ and there exists a bounded sequence $\left\{J_{n}\right\}_{n=0}^{\infty}$ of nonzero operators in $\mathscr{L}(\mathscr{H})$ such that $J_{0}$ is compact and either

(a) $J_{n-1} Q=Q J_{n}, n \in \mathbb{N}$ or

(b) $Q J_{n-1}=J_{n} Q, n \in \mathbb{N}$

(which is obviously the case if there is a nonzero compact operator in $\left.(Q\}^{\prime}\right)$. Then $Q$ has a nontrivial hyperinvariant subspace. 
PROOF. Without loss of generality we may suppose that $Q$ is a quasiaffinity and that each $J_{n-1}, n \in \mathbb{N}$, is a contraction. Moreover, as is well known, to show that $Q$ has a nontrivial hyperinvariant subspace, it suffices to show that $Q^{*}$ does, so in case (b) holds we take adjoints and obtain instead that (a) holds. Apply Corollary 3.3 (see, for example, (8)) with $C:=J_{0}$ to obtain the sequences $\left\{n_{k}\right\},\left\{y_{n_{k}}\right\}$, and $\left\{z_{k}\right\}$ with the properties set forth there. Let $S$ be an arbitrary nonzero contraction in $\{Q\}^{\prime}$, and define $A_{k, m}=S J_{n_{k}}, k, m \in \mathbb{N}$. Then, after defining

$$
\begin{array}{rlrl}
\beta_{k} & =\left\|y_{n_{k}}\right\|\left\|\left(Q^{n_{k}+1}\right)^{*}\left(z_{k}-x_{0}\right)\right\|, & & s_{k}=Q^{n_{k}} y_{n_{k}}, \\
t_{k} & =Q^{*}\left(z_{k}-x_{0}\right), \quad k \in \mathbb{N}, \quad \text { and } \quad t_{0}=Q^{*}\left(z_{0}-x_{0}\right) \neq 0,
\end{array}
$$

we obtain from (8) that

$$
\begin{aligned}
\left|\left(S J_{0} s_{k}, t_{k}\right)\right| & =\left|\left(S J_{0} Q^{n_{k}} y_{n_{k}}, t_{k}\right)\right|=\left|\left(S Q^{n_{k}} J_{n_{k}} y_{n_{k}}, t_{k}\right)\right| \\
& =\left|\left(S J_{n_{k}} y_{n_{k}},\left(Q^{n_{k}}\right)^{*} t_{k}\right)\right| \leq \beta_{k}, \quad k \in \mathbb{N} .
\end{aligned}
$$

Since $\left\|S J_{0} s_{k}-S J_{0} s_{0}\right\| \rightarrow 0$ (this uses the compactness of $J_{0}$ ), and $\left\{t_{k}\right\}_{k \in \mathbb{N}}$ converges weakly to $t_{0} \neq 0$, we obtain $\left(S J_{0} s_{0}, t_{0}\right)=0$. Since $J_{0} s_{0} \neq 0$ from above, and $S$ is an arbitrary nonzero contraction in $\{Q\}^{\prime}$, this shows that $\left(\{Q\}^{\prime} J_{0} s_{0}\right)^{-}$is a nontrivial hyperinvariant subspace for $Q$.

Along similar lines we obtain the following improvement (in the quasinilpotent case) of Lomonosov's theorem [6] about operators whose commutant has the 'PearcySalinas property'.

COROLlARY 3.5. Suppose that $Q \neq 0$ is a quasinilpotent operator in $\mathscr{L}(\mathscr{H})$ and there exist bounded sequences $\left\{J_{m}\right\}_{m \in \mathbb{N}},\left\{L_{m}\right\}_{m \in \mathbb{N}}$, and $\left\{K_{n}\right\}_{n \in \mathbb{N}}$ in $\mathscr{L}(\mathscr{H})$ such that $\left\{K_{n}\right\} \subset \mathbb{K},\left\|L_{m}-K_{m}\right\| \rightarrow 0, Q^{n} J_{m}=L_{m} Q^{n}$ for all $m, n \in \mathbb{N}$, and $\left\{L_{m}\right\}$ converges in the weak operator topology to an operator $R \neq 0$. Then $Q$ has a nontrivial hyperinvariant subspace.

PROOF. We may suppose, without loss of generality, that $Q$ is a quasiaffinity and that the sequences $\left\{J_{m}\right\},\left\{K_{m}\right\}$, and $\left\{L_{m}\right\}$ are all contractions. We apply Theorem 3.1 and Corollary 3.3 (especially (8)) to $Q$ with $C:=R$ to obtain the sequences $\left\{s_{k}\right\},\left\{t_{k}\right\}$, and $\left\{\beta_{k}\right\}$ defined in Corollary 3.4. Let now $S$ be an arbitrary nonzero contraction in $\{Q\}^{\prime}$. We will show that $\left(S R s_{0}, t_{0}\right)=0$, and therefore that $\left(\{Q\}^{\prime} R s_{0}\right)^{-}$is the desired nontrivial hyperinvariant subspace for $Q$. (Note that $R s_{0}$ and $t_{0}\left(=\mathrm{w}-\lim t_{k}\right)$ are nonzero by Theorem 3.1.) We define the doubly indexed sequence $\left\{A_{k, m}\right\}$ of (8) of Corollary 3.3 by $A_{k, m}=S J_{m}, k, m \in \mathbb{N}$, and thus obtain

$$
\left|\left(S L_{m} s_{k}, t_{k}\right)\right|=\left|\left(Q^{n_{k}} S J_{m} y_{n_{k}}, t_{k}\right)\right| \leq \beta_{k}, \quad k, m \in \mathbb{N} .
$$


Let $\eta>0$ be given and note that (since $\left\{K_{m}\right\}$ tends to $R$ in the weak operator topology) it suffices to find $M_{\eta} \in \mathbb{N}$ such that

$$
\left|\left(S K_{m} s_{0}, t_{0}\right)\right| \leq \eta, \quad m \geq M_{\eta} .
$$

Choose $M_{\beta} \in \mathbb{N}$ such that for $k \geq M_{\beta}, \beta_{k}<\eta / 2$, and choose $M_{\eta} \in \mathbb{N}$ such that

$$
\left\|L_{m}-K_{m}\right\|<\frac{\eta}{2\|S\|\left(\sup _{k}\left\|s_{k}\right\|\left\|t_{k}\right\|\right)}, \quad m \geq M_{\eta} .
$$

Then, via (10) and (12), we have, for $m \geq M_{\eta}$ and $k \geq M_{\beta}$,

$$
\left|\left(S K_{m} s_{k}, t_{k}\right)\right| \leq\left|\left(S L_{m} s_{k}, t_{k}\right)\right|+\left|\left(S\left(K_{m}-L_{m}\right) s_{k}, t_{k}\right)\right|<\eta .
$$

Fix an arbitrary integer $m_{0} \geq M_{\eta}$ and note that since $\left\{s_{k}\right\}$ tends weakly to $s_{0}$ and $S K_{m_{0}}$ is compact,

$$
\lim _{k}\left\|S K_{m_{0}} s_{k}-S K_{m_{0}} s_{0}\right\|=0 .
$$

From (13), (14), and the fact that $\left\{t_{k}\right\}$ tends weakly to $t_{0}$, we obtain

$$
\left|\left(S K_{m_{0}} s_{0}, t_{0}\right)\right|=\lim _{k}\left|\left(S K_{m_{0}} s_{k}, t_{k}\right)\right| \leq \eta, \quad m_{0} \geq M_{\eta},
$$

which establishes (11) and completes the proof.

The following lemma is elementary and needs no proof.

LEMMA 3.6. Suppose $\left\{p_{n}\right\}_{n=1}^{\infty}$ is a sequence of positive numbers such that

$$
\lim _{n}\left(p_{n}\right)^{1 / n}=0
$$

Then there exists a subsequence $\left\{p_{n_{k}}\right\}_{k=1}^{\infty}$ of $\left\{p_{n}\right\}$ such that $\lim _{k}\left(p_{n_{k}+1} / p_{n_{k}}\right)=0$.

The next result is a structure theorem for quasinilpotent operators, and has some interesting consequences. Its discovery was motivated by the desire to use (9) of Corollary 3.3 to good advantage. Our first proof of it used Corollary 3.3, and only later did we find the elementary proof below.

THEOREM 3.7. Suppose $Q$ is a nonnilpotent quasinilpotent operator in $\mathscr{L}(\mathscr{H})$ and there exists some bounded sequence $\left\{X_{n}\right\}_{n \in \mathrm{N}}$ in $\mathscr{L}(\mathscr{H})$ such that either the sequence $\left\{\left(Q^{n} /\left\|Q^{n}\right\|\right) X_{n}\right\}$ or the sequence $\left\{X_{n} Q^{n} /\left\|Q^{n}\right\|\right\}$ converges in the weak operator topology to a nonzero operator $R$. Then $Q$ is not a quasiaffinity and thus has a nontrivial hyperinvariant subspace. 
PROOF. We suppose that the sequence $\left\{\left(Q^{n} /\left\|Q^{n}\right\|\right) X_{n}\right\}$ converges to $R \neq 0$ in the weak operator topology and show that $Q R=0$. The other assertion is proved by taking adjoints. Consider the sequence $\left(Q^{n+1} /\left\|Q^{n}\right\|\right) X_{n}$, which obviously converges to $Q R$ in the weak operator topology. By Lemma 3.6 there exists a subsequence $\left\{Q^{n_{k}}\right\}_{k=1}^{\infty}$ of $\left\{Q^{n}\right\}$ such that the sequence $\left\{\left\|Q^{n_{k}+1}\right\| /\left\|Q^{n_{k}}\right\|\right\}$ converges to zero. Thus,

$$
\left(Q^{n_{k}+1} /\left\|Q^{n_{k}}\right\|\right) X_{n_{k}}=\left(\left\|Q^{n_{k}+1}\right\| /\left\|Q^{n_{k}}\right\|\right)\left(Q^{n_{k}+1} /\left\|Q^{n_{k}+1}\right\|\right) X_{n_{k}}, \quad k \in \mathbb{N},
$$

and since the first term in the product on the right side of (15) tends to zero and the second term is bounded, we get from (15) that $Q R=0$, as desired.

This yields immediately the following results.

COROLlARY 3.8. Suppose that $Q$ is a nonnilpotent quasinilpotent operator in $\mathscr{L}(\mathscr{H})$ and there exists a subspace $\mathscr{M} \neq(0)$ such that the sequence of restrictions $\left\{\left.\left[Q^{n}\left(Q^{*}\right)^{n} /\left\|Q^{n}\right\|^{2}\right]\right|_{\mathscr{M}}\right\}$ (mapping $\mathscr{M}$ into $\mathscr{H}$ ) converges in the weak operator topology to a nonzero operator. Then $Q$ has a nontrivial hyperinvariant subspace.

Proof. Let $E$ be the projection in $\mathscr{L}(\mathscr{H})$ whose range is $\mathscr{M}$. The hypotheses yield immediately that the sequence $\left\{\left(Q^{n} /\left\|Q^{n}\right\|\right)\left(\left(Q^{*}\right)^{n} /\left\|Q^{n}\right\|\right) E\right\}$ converges (weakly) to a nonzero operator, and the result follows from Theorem 3.7.

COROLlaRY 3.9. Suppose that $Q$ is a nonnilpotent quasinilpotent operator in $\mathscr{L}(\mathscr{H})$ such that the sequence $\left\{Q^{n}\left(Q^{*}\right)^{n} /\left\|Q^{n}\right\|^{2}\right\}$ of positive operators has a common eigenvector $e_{0}$ with corresponding (nonnegative) eigenvalues $\left\{\lambda_{n}\right\}(\leq 1)$ such that $\lim _{n} \lambda_{n}$ exists and is nonzero. Then $Q$ has a nontrivial hyperinvariant subspace.

\section{Some problems}

We close this note with what seem to be some interesting problems about quasinilpotent operators and their invariant subspaces.

(1) Generalize Corollary 3.9 by showing that if $Q$ is a quasinilpotent operator in $\mathscr{L}(\mathscr{H})$ such that the operators in the sequence $\left\{Q^{n}\left(Q^{*}\right)^{n}\right\}_{n \in \mathbb{N}}$ have a common eigenvector, then $Q$ has a nontrivial hyperinvariant subspace.

(2) Show that if $Q$ is a quasinilpotent operator in $\mathscr{L}(\mathscr{H})$ such that the sequence $\left\{Q^{n}\left(Q^{*}\right)^{n}\right\}_{n \in \mathrm{N}}$ consists of mutually commuting operators, then $Q$ has a nontrivial hyperinvariant subspace.

(3) It is known that if $Q$ is an arbitrary quasinilpotent operator in $\mathscr{L}(\mathscr{H})$, then there exist compact operators $K_{1}$ and $K_{2}$ and quasiaffinities $X$ and $Y$ in $\mathscr{L}(\mathscr{H})$ such that $Q X=X K_{1}$ and $Y Q=K_{2} Y$ [3]. (It is also known that not every quasinilpotent 
operator in $\mathscr{L}(\mathscr{H})$ is quasisimilar to a compact operator [3].) Use these facts together with Corollary 3.3 to show that every quasinilpotent operator in $\mathscr{L}(\mathscr{H})$ has a nontrivial hyperinvariant subspace.

\section{Acknowledgement}

This research was supported by a grant from the Korea Research Foundation (KRF 2002-070-C00006).

\section{References}

[1] S. Ansari and P. Enflo, 'Extremal vectors and invariant subspaces', Trans. Amer. Math. Soc. 350 (1998), 539-558.

[2] H. Bercovici, C. Foiaş and C. Pearcy, Dual algebras with applications to invariant subspaces and dilation theory, CBMS Regional Conference Series in Mathematics 56 (Amer. Math. Soc., Providence, RI, Published for the Conference Board of the Mathematical Sciences, Washington, DC, 1985).

[3] C. Foiaş and C. Pearcy, 'A model for quasinilpotent operators', Michigan Math. J. 21 (1974), 399-404.

[4] I. Jung, E. Ko and C. Pearcy, 'On quasinilpotent operators', Proc. Amer. Math. Soc. 131 (2003), 2121-2127.

[5] V. Lomonosov, 'On invariant subspaces of families of operators commuting with a completely continuous operator', Funktsional. Anal. i Prilozen. 7 (1973), 55-56.

[6] - 'An extension of Burnside's theorem to infinite dimensional spaces', Israel J. Math. 75 (1991), 329-339.

[7] C. Pearcy and N. Salinas, 'An invariant subspace theorem', Michigan Math. J. 20 (1973), 21-31.

[8] C. Pearcy and A. Shields, 'A survey of the Lomonosov technique in the theory of invariant subspaces', in: Topics in operator theory, Math. Surveys 13 (Amer. Math. Soc., Providence, RI, 1974) pp. 219 230.

Department of Mathematics

Texas A\&M University

College Station

TX 77843

USA

e-mail: foias@math.tamu.edu
Department of Mathematics College of Natural Science Kyungpook National University Daegu 702-701

Korea e-mail: ibjung@knu.ac.kr
Department of Mathematics

Ewha Women's University

Seoul 120-750

Korea

e-mail: eiko@ewha.ac.kr
Department of Mathematics

Texas A\&M University

College Station

TX 77843

USA

e-mail: pearcy@math.tamu.edu 